\title{
Erratum to: Bacteria-induced hatching of Trichuris muris eggs occurs without direct contact between eggs and bacteria
}

\section{Koichi Koyama $^{1}$}

Published online: 20 February 2016

(C) Springer-Verlag Berlin Heidelberg 2016

\section{Erratum to: Parasitology Research}

\section{DOI 10.1007/s00436-015-4795-2}

The original version of the above article contained a mistake in Figure 2b, incubation time. It should be the same as Figure $2 \mathrm{a}$. The corrected figure is given below.
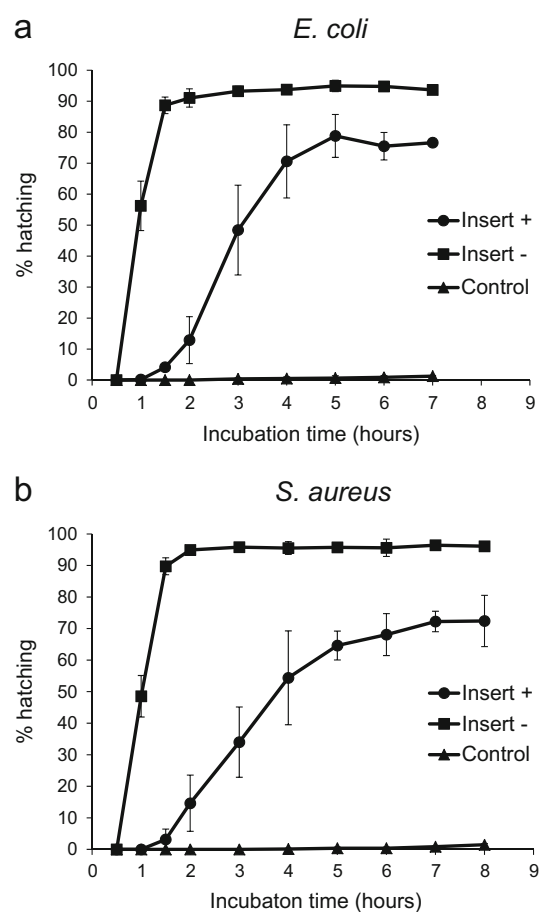

The online version of the original article can be found at http://dx.doi.org/ 10.1007/s00436-015-4795-2.

\section{Koichi Koyama}

koyama@kitasato-u.ac.jp

1 Department of Parasitology, Kitasato University School of Medicine, 1-15-1 Kitasato, Minami, Sagamihara, Kanagawa 252-0374, Japan 